\title{
Lidil
}

Revue de linguistique et de didactique des langues

$50 \mid 2014$

Variation stylistique et diversité des contextes de socialisation

\section{Jean-Luc Dorier, Francia Leutenegger et Bernard Schneuwly (dir.), Didactique en construction, construction des didactiques}

Bruxelles, De Boeck Supérieur, collection de la Section des sciences de l'éducation de l'université de Genève, 2013

\section{Sandra Canelas-Trevisi}

\section{OpenEdition \\ Journals}

\section{Édition électronique}

URL : http://journals.openedition.org/lidil/3663

DOI : $10.4000 /$ lidil.3663

ISSN : $1960-6052$

\section{Éditeur}

UGA Éditions/Université Grenoble Alpes

\section{Édition imprimée}

Date de publication : 15 décembre 2014

Pagination : 217-219

ISBN : 978-2-84310-287-5

ISSN : $1146-6480$

\section{Référence électronique}

Sandra Canelas-Trevisi, « Jean-Luc Dorier, Francia Leutenegger et Bernard Schneuwly (dir.), Didactique en construction, construction des didactiques », Lidil [En ligne], 50 | 2014, mis en ligne le 15 juin 2016, consulté le 25 septembre 2020. URL : http://journals.openedition.org/lidil/3663 ; DOI : https://doi.org/ 10.4000/lidil.3663 
de savoir, l'ethnotype parisien représentant la norme, et la variation sociolinguistique symbolisée par les Belges. Puis M. Causa insiste sur l'importance des notions de discours, de texte et de genre dans le contexte de l'enseignement d'une discipline non linguistique en L2. Elle prône une approche de didactique intégrée, demandant une «pédagogie de projet» qui vise une collaboration de l'équipe autour de l'amélioration des compétences linguistiques, disciplinaires et didactiques.

L'ouvrage se termine avec une postface de S. Moirand, qui retrace brièvement l'historique du CEDISCOR, avant de tisser les liens entre les contributions de ce numéro. D'abord, l'hybridité discursive se manifeste lors des points de contact, via les technologies discursives et autres moyens, entre experts, professionnels et citoyens. Elle propose d'approfondir la notion de genre de discours, qui met en cause le concept d'une transmission purement linéaire de l'expert vers le non-expert. Ensuite, elle souligne l'importance de la visée pragmatique du discours. Elle conclut avec une série de questions qui promet d'autres recherches dynamiques autour de l'évolution de configurations discursives.

Laura Hartwell

Université Grenoble Alpes, LIDILEM

Jean-Luc Dorier, Francia Leutenegger et Bernard Schneuwly
(dir.), Didactique en construction, construction des didactiques, Bruxelles, De Boeck Supérieur, collection de la Section des sciences de l'éducation de l'université de Genève, 2013

Cette livraison de la collection «Raisons éducatives» porte un regard pluriel sur deux questions : est-il possible de cerner les coordonnées d'un champ disciplinaire qui s'appellerait «la didactique»? Comment les didactiques des disciplines se placent-elles dans ce champ? Dans l'introduction, les éditeurs se proposent d'identifier les principes, les hypothèses et les démarches susceptibles de fédérer les didactiques des disciplines et, inversement, les spécificités de chacune d'entre elles ainsi que les obstacles potentiels à la construction d'un champ disciplinaire «didactique». Pour les éditeurs, les projets des didactiques des disciplines, malgré leur relative hétérogénéité, comportent tous des ingrédients, certains développés, d'autres en devenir, qui rendent possible 
la construction de la didactique, comme «champ scientifique dédié à la compréhension de la transmission et de la diffusion des savoirs dans la société» (p. 7). L'introduction, particulièrement riche et éclairante, développe, après un aperçu historique, les aspects susceptibles de délimiter et de structurer le champ de «la didactique», sans oublier pour autant «les tensions séparatistes». Sont évoqués : a) le caractère particulier de la disciplinarisation de la didactique, qui s'est construite en référence à un champ professionnel et social préexistant, à savoir les métiers de l'enseignement, et se différencie ainsi d'autres disciplines organisées plutôt selon une logique de développement interne, orientée par des questions de savoirs; b) les éléments de théorisation partagés, comme les débats théoriques autour de la transposition didactique ou ceux liés à l'observation et à l'analyse des classes dites ordinaires, courant de recherches initié par la didactique comparée et pourvoyeur d'outils théoriques reconfigurés ensuite dans les recherches des didactiques disciplinaires.

Le programme de promouvoir la construction du champ de «la didactique» se concrétise dans l'ouvrage à l'aide de 12 contributions. Les deux premières s'intéressent au développement de l'idée didactique dans l'Europe continentale, l'une illustre les spécificités de la didactique disciplinaire en Allemagne, l'autre les recherches en didactique et le comparatisme en France.

Les 10 contributions qui suivent émanent toutes des équipes des didactiques disciplinaires et comparée de l'université de Genève et de leurs collaborateurs, en Europe et en Amérique du Sud. Les textes se répartissent selon deux axes : les cinq premiers sont centrés sur l'ancrage social et historique des contenus disciplinaires; les autres portent sur les concepts et les méthodes en didactique(s).

Dans les textes du premier axe, les contenus disciplinaires sont interrogés du point de vue des savoirs de référence et de la demande sociale. Le premier texte porte sur la corporéité dans les didactiques des arts (danse, théâtre, musique, arts visuels) et sur la construction sociale et culturelle de la corporéité à l'école. Le deuxième, adoptant un point de vue institutionnel, illustre la difficulté de décrire la discipline scolaire «éducation physique» à l'école primaire et s'intéresse, entre autres, à l'articulation de l'action didactique respective du maitre spécialiste de la discipline, qui intervient en classe une fois toutes les deux semaines, et du maitre généraliste. Les deux textes suivants, consacrés l'un à la didactique de la géographie, l'autre à celle de l'histoire, mettent l'accent sur la spécificité des savoirs de référence et sur le dialogue complexe 
entre les deux disciplines et le monde social. Pour réagir à la doxa qui «tyrannise l'histoire scolaire», une «grammaire du questionnement scolaire de l'histoire» est proposée. Le dernier texte du premier axe illustre les pratiques d'interprétation des œuvres littéraires au collège genevois (équivalent du lycée) et leurs implications.

Les textes du second axe mettent à l'épreuve les concepts et les méthodes des didactiques. Le premier s'intéresse à l'École de culture générale (enseignement secondaire post-obligatoire genevois) et s'interroge sur l'impact de l'introduction dans le curriculum de l'argumentation en français et en physique. Le second texte porte sur les objets d'enseignement en français et sur les méthodes pour les appréhender en contexte de classe d'une part, de formation d'enseignants d'autre part. Le suivant s'intéresse aux tâches, aux exercices et aux problèmes dans trois disciplines (langue étrangère, mathématiques et sciences), avec l'objectif de cerner la culture disciplinaire de chacune d'entre elles. L'avant-dernier texte aborde la fonction didactique de la justification en lecture/compréhension et en sciences de la nature. Cette étude s'inscrit dans le programme pluridisciplinaire en didactique du Réseau Maison des petits, à Genève. Enfin, le dernier texte porte sur la diversité des approches en didactique des mathématiques dans l'enseignement primaire en Suisse romande.

Sandra Canelas-Trevisi Université Grenoble Alpes, LIDILEM et GRAFE

Semen, $n^{\circ}$ 36, «Les nouveaux discours publicitaires», nov. 2013

Neuf contributions (France, Suisse, Italie, Chili) coordonnées par M. Bonhomme font le point sur les modalités d'inscription de la publicité. On pourrait parcourir les articles proposés avec le prisme du masquage/ dévoilement ou de l'effacement/déplacement des trois «objets» de la publicité : produit, consommateur, annonceur. M. Bonhomme analyse la plasticité du discours publicitaire (d.p.) s'adaptant à son environnement technique et social, pointant la perspective interactive affichée des nouveaux messages publicitaires. J.-C. Soulages cible, lui, les imaginaires («images de la réalité, mais en tant que cette image interprète la réalité, la fait entrer dans un univers de signification », Charaudeau, 2005 , p. 158) et ce qu'ils disent d'un produit devenu un bienfait, d'un 